\title{
Characterizing the galaxy populations within different environments in the RCS2319 supercluster
}

\author{
Anna Delahaye and Tracy Webb \\ Department of Physics, McGill University, \\ 3600 rue University, H3A 2T8, Montreal Quebec, Canada \\ email: delahaye@physics.mcgill.ca
}

\begin{abstract}
We present the results of a multi-wavelength photometric study of the high redshift supercluster RCS2319+00. RCS2319+00 is a high-redshift $(z \sim 0.9)$ supercluster comprising three spectroscopically confrmed cluster cores discovered in the Red Sequence Cluster Survey (RCS) (Gladders \& Yee 2005). Core proximities and merger rates estimate coalescence into a $10^{15} M_{\odot}$ cluster by $z \sim 0.5$ (Gilbank et al. 2008). Spectroscopic studies of the system have revealed over 300 supercluster members located in the cores and several infalling groups (Faloon et al. 2013). RCS2319 presents a diverse range of dynamical systems and densities making it an ideal laboratory in which to study the effects of environment on galaxy properties.

Imaging in optical and near infrared ( $g r i z^{\prime}$ from MegaCam, $J K_{s}$ from WIRCam, both at CFHT), as well as $3.6 \mu \mathrm{m}$ and $4.5 \mu \mathrm{m}$ from IRAC have enabled the assembly of a large photometric catalogue. Coupled with an extensive spectroscopic survey (Faloon et al. 2013) providing nearly 2400 redshifts across the field, photometric redshifts were determined using the template fitting code EAZY (Brammer et al. 2008). Nearly 80000 photometric redshifts were measured providing a sample of nearly 3000 cluster members.

To investigate effects of global environment, analysis was done utilizing a friend-of-friends group finding algorithm identifying several large and small infalling groups along with the three cluster cores. The cores are found to be dominated by massive, red galaxies and the field galaxies are populated by low mass, blue galaxies, as is the case in the local universe. Interestingly, the large groups exhibit intermediate properties between field and core populations, suggesting possible pre-processing as they are being accreted into the core halos.

Relative fifth-nearest neighbour overdensity, $\log \left(1+\delta_{5}\right)$, is used as a proxy for local environment to investigate environmental dependence on galaxy colour. While there is an overall dependence of colour on local density, when controlled for stellar mass the dependence largely disappears. Indeed, galaxy mass is the dominant factor in determining colour, with local density a secondary effect only noticeable in lower mass galaxies at the $3 \sigma$ level for both colour and red fraction.

RCS2319+00 presents a rare opportunity to probe many different densities and environments all located within the same object. We're able to investigate how galaxy evolution is affected by the environment, from field galaxies to infalling to groups to dense cluster cores, as well as the different density regions within each environment.
\end{abstract}

Keywords. Galaxy Clusters, Galaxies: photometry, Galaxy Environments

\section{References}

Brammer, G. B., van Dokkum, P. G., \& Coppi, P. 2008, ApJ, 686, 1503

Faloon, A. J., Webb, T. M. A., Ellingson, E., et al. 2013, ApJ, 768, 104

Gilbank, D., Yee, H. K. C., Ellingson, E., et al. 2008, ApJ, 677, L89

Gladders, M. \& Yee, H. 2005, ApJS, 157, 1 